\title{
Sarcocystis species in wild and domestic sheep (Ovis ammon and Ovis aries) from China
}

\author{
Hui Dong ${ }^{1}$, Ruijing Su${ }^{1}$, Yinghua Wang ${ }^{2}$, Zongxi Tong ${ }^{1}$, Longxian Zhang ${ }^{1}$, Yurong Yang ${ }^{1 *}$ (D) and Junjie $\mathrm{Hu}^{3^{*}}$
}

\begin{abstract}
Background: Sarcocystis species are intracellular protozoan parasites that can pose a threat to animal health and food safety. The aim of this study was to investigate the prevalence of infection with Sarcocystis infection in sheep from China.

Results: In total, 52.51\% (335/638) of tissue samples from domestic sheep contained sarcocysts through examination by light microscopy. The organisms were identified as $S$. tenella and S. arieticanis by molecular assays. Macroscopic S. gigantea and S. medusiformis were not found. The average sarcocysts loading was $18.07 \pm 29.87$ per square centimeter in the myocardium of domestic sheep. Furthermore, two specimens of argali (Ovis ammon) were examined and sarcocysts were found in the myocardium of one animal. According to the sequence of the cox1 gene of sarcocysts from argali, it was speculated as S. tenella.

Conclusions: We found a high prevalence and parasite load of Sarcocystis in sheep from both central and northwest China. This report is the first to indicate that argali may be a natural intermediate host for $\mathrm{S}$. tenella.
\end{abstract}

Keywords: Sarcocystis, Argali, Sheep, Parasite load, S. tenella, S. arieticanis, China

\section{Background}

Sarcocystis are intracellular protozoan and food-borne parasites [1]. Currently, 196 valid Sarcocystis species are recognized [2], each of which has a strict host and genus specificity. The parasites form cysts that are found in the striated muscles and central nervous system of livestock, such as swine, cattle, and sheep [2,3]. Human can be infected with S. hominis, S. heydorni, and S. suihominis by consumption of undercooked meat containing the sarcocysts [2, 4]. The European Food Safety Authority has classified S. hominis and S. suihominis as zoonotic hazards for which official meat inspections need to be vigilant [5]. Sarcocystosis contributes to weight loss, abortion, premature birth, and even death in sheep; these animals are usually infected by ingesting water and by foraging for feed contaminated with Sarcocystis sporocysts [2].

\footnotetext{
* Correspondence: yangyu7712@sina.com; jjhu@ynu.edu.cn

${ }^{1}$ College of Animal Science and Veterinary Medicine, Henan Agricultural University, Zhengzhou 450002, China

${ }^{3}$ School of Biological Sciences, Yunnan University, Kunming 650091, China Full list of author information is available at the end of the article
}

Most Sarcocystis species have an obligatory two-host life cycle, with carnivores as definitive hosts and herbivores as intermediate hosts [6]. There have been only four validated species described in sheep: the non-pathogenic macroscopic $S$. gigantea and $S$. medusiformis transmitted by felids and the pathogenic microscopic $S$. tenella and $S$. arieticanis transmitted by canids [2]. S. tenella, S. arieticanis, and S. gigantea are distributed worldwide, including China. However, S. medusiformis has only been reported from Italy, Iran, New Zealand, Spain, Jordan and Australia [2,7].

In China, the annual production of meat from sheep in 2017 was 4851 thousand tonnes. The average prevalence of Sarcocystis infection in sheep was $41.52 \%$ $(14,639 / 35254)$ in China [3], which was only slightly lower than that of the entire world $(46.72 \%, 32,314 /$ 69158) [2]. Sarcocystis infection can cause economic losses in animal husbandry and represents a threat to public health and food safety [5]. However, there are few reports available in Chinese journals concerning sarcocystosis in sheep. In most studies, detection was done by naked eye and muscle squash. Further, studies of 
sarcocystosis in wildlife may provide a point of comparison for understanding the evolution and development of the parasite in domestic animals in human habitats. Argali (Ovis ammon) belongs to order Artiodactyla, suborder Ruminantia, infraorder Pecora, family Bovidae, subfamily Caprinae, tribe Caprini, genus Ovis. In the present work, we examined the prevalence, morphology, and molecular characteristics of Sarcocystis species in the muscles of domestic sheep and argalis from China.

\section{Results}

Macroscopic sarcocysts were not found in 640 sheep hearts. The prevalence of infection with Sarcocystis species in the domestic sheep was 52.51\% (335/638) (Table 1), and sarcocysts were detected in the myocardium of one argali out of two animals inspected by histological examination.

Thick-walled cysts of S. tenella and thin-walled cysts of S. arieticanis were observed in the samples by light microscopy (Fig. 1a, b). The proportion of thick-walled sarcocysts was higher than that of thin-walled sarcocysts $(P$ $<0.05)$. The average sarcocyst loading was $18.07 \pm 29.87$ per square centimeter; the tissue with the highest number of sarcocysts observed $(n=302)$ is shown in Fig. 1c. Compared to the fraction of samples with less than 10 sarcocysts $/ \mathrm{cm}^{2}$ was $201 / 335$ (60.00\%); 10-50 sarcocysts $/ \mathrm{cm}^{2}$ were present in $95 / 335(28.36 \%)$ of the samples $(P<0.01)$; and more than 50 sarcocysts $/ \mathrm{cm}^{2}$ were present in $39 / 335$ (11.64\%) of the samples $(P<0.01)$. We observed inflammatory cells around the sarcocysts, indicating myositis, in $3.45 \%(22 / 638)$ of the samples (Fig. 1d).
Under light microscopy, the sarcocysts appeared fusiform or oval-shaped; they were not Toxoplasma gondii cysts, which confirmed by IHC-staining (Fig. 1e). Sarcocysts were also observed in Purkinje fibers (Fig. 1f). Immature sarcocysts with numerous metrocytes were found in the myocardium from domestic sheep (Fig. 1g). The size of $S$. arieticanis cysts was $6.51-105.00 \mu \mathrm{m} \times 6.46-37.63 \mu \mathrm{m}$ $(n=40)$; villar protrusions were hair-like; the walls of the cysts were $0.24 \pm 0.23 \mu \mathrm{m}$ thick, and the size of the bradyzoites was $2.68-6.27 \mu \mathrm{m} \times 0.9-1.89 \mu \mathrm{m}(n=20)$. For S. tenella, the size of cysts was $15.20-88.00 \mu \mathrm{m} \times 11.19$ $33.11 \mu \mathrm{m}(\mathrm{n}=40)$; the villar protrusions were finger-like (Fig. 1h); the wall was $0.56 \pm 0.54 \mu \mathrm{m}$ thick, and bradyzoites measured $3.56-5.62 \mu \mathrm{m} \times 1.21-1.84 \mu \mathrm{m} \quad(n=20)$. The sarcocysts contained crescent-shaped bradyzoites located in numerous chambers formed by septa (Fig. 1h, i). We also found sarcocysts in the myocardium of argali: the size of the cysts was $16.74-17.83 \mu \mathrm{m} \times 55.23-$ $61.74 \mu \mathrm{m}(n=3)$; the villar protrusions were finger-like, and the wall was $0.13 \pm 0.09 \mu \mathrm{m}$ thick (Fig. 1j). The morphology of sarcocysts found in argali mostly resembled that of the $S$. tenella cysts in domestic sheep.

Variation associated with the risk factors of location and season of the year is shown in Table 2. The locations of the provinces where the samples were collected showed no correlation with Sarcocystis prevalence $(P>0.05)$. The risk of Sarcocystis infection in the autumn was higher than that in the spring and winter $(P<0.01)$.

The cox 1 amplification and sequence analysis verified that sarcocysts from argali were S. tenella (Fig. 2). Furthermore, the mitochondrial cox 1 nucleotide sequences from sarcocysts of argali were submitted to GenBank (accession number

Table 1 Infection rate and loading of Sarcocystis species in domestic sheep

\begin{tabular}{|c|c|c|c|c|c|c|c|}
\hline Batch number & Location $^{\text {a }}$ & City & Sample received date & No. of samples & No. of positives & Infection rate (\%) & Density (cysts/ $\mathrm{cm}^{2}$ ) \\
\hline 1 & I & Zhengzhou & 1 Mar 2014 & 155 hearts & 20 & 12.90 & 6.35 \\
\hline 2 & ॥ & Luoyang & 18 Oct 2015 & 32 hearts & 10 & 31.25 & 22.53 \\
\hline 3 & III & Xinxiang & 20 Nov 2016 & 10 hearts & 0 & - & - \\
\hline 4 & \multirow[t]{4}{*}{ IV $(n=254)$} & \multirow[t]{4}{*}{ Jiaozuo } & 9 Nov 2015 & 70 hearts & 62 & \multirow{4}{*}{$\begin{array}{l}93.70 \\
(238 / 254)\end{array}$} & 26.96 \\
\hline 5 & & & 25 Nov 2016 & 58 hearts & 58 & & 18.73 \\
\hline 6 & & & 16 Oct 2017 & 62 hearts & 55 & & 19.73 \\
\hline 7 & & & 19 Oct 2017 & 64 hearts & 63 & & 22.65 \\
\hline 9 & \multirow[t]{4}{*}{$V(n=82)$} & \multirow[t]{4}{*}{ Zhumadian } & 8 Oct 2015 & 15 hearts & 3 & \multirow{4}{*}{$\begin{array}{l}10.98 \\
(9 / 82)\end{array}$} & 0.56 \\
\hline 10 & & & 30 Nov 2015 & 36 hearts & 3 & & 12.44 \\
\hline 11 & & & 1 Dec 2015 & 20 hearts & 2 & & 0.33 \\
\hline 12 & & & 7 Dec 2015 & 11 hearts & 1 & & 0.33 \\
\hline 13 & $\mathrm{VI}$ & Xinyang & 8 Jul 2015 & 3 hearts & 0 & - & - \\
\hline 14 & VII & Hami & 4 Jan 2016 & 102 hearts & 58 & 56.86 & 6.60 \\
\hline 15 & Total & & & 638 hearts & 335 & 52.51 & 18.07 \\
\hline
\end{tabular}

${ }^{\text {a Sampling areas in Fig. } 1 .}$ 

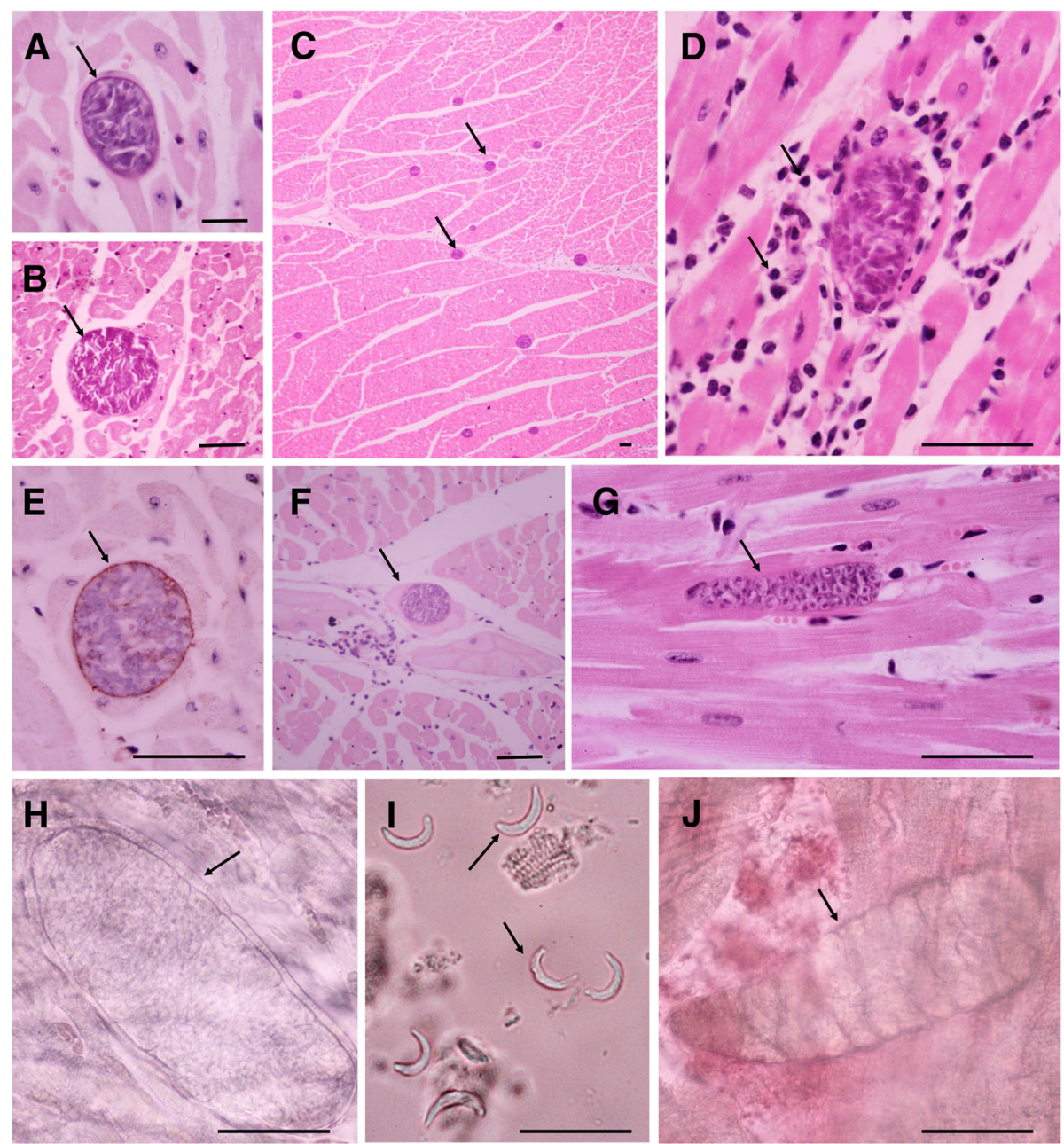

Fig. 1 Sarcocystis infection in the myocardium of sheep. LM, H\&E. Bar $=50 \mu \mathrm{m}$. a Thick-walled S. tenella sarcocyst, domestic sheep. $\mathbf{b}$ Thin-walled S. arieticanis sarcocyst, domestic sheep. c Numerous sarcocysts in sheep myocardium, domestic sheep. $\mathbf{d}$ Inflammatory cells infiltrating the area around a sarcocyst, domestic sheep. e Cysts testing negative for Toxoplasma gondii, domestic sheep. f A sarcocyst in Purkinje fibers, domestic sheep. $\mathbf{g}$ Immature sarcocyst with numerous metrocytes, domestic sheep. $\mathbf{h}$ Bradyzoites are located in the numerous chambers, created by the septa of the sarcocyst; the finger-like villar protrusions around a cyst wall; myocardium squash, unstained, domestic sheep. i Bradyzoites in pepsin-digestion liquid; unstained, domestic sheep. j Sarcocyst from myocardium squash, unstained, argali

MH561854). The sequence was approximately $989 \mathrm{bp}$ in length and had high identity with $S$. tenalla. The sequence showed 99\% identity with those of S. tenalla (KC209723KC209732) (KP263746-KP263751) from Norwegian sheep and Polish Tatra chamois, respectively. Additionally, there was $97 \%$ identity with S. tenalla (MF039322 and MF039323) from sheep, 93\% identity with S. capracanis (KU820974 and KU820977) from goats, and 90\% identity with S. heydorni (KX057994 and KX057995) from cattle in China. The phylogenetic analysis based on near full-length mitochondrial cox1

Table 2 Odds ratio of province and season as risk factors for the prevalence of infection with Sarcocystis species in sheep

\begin{tabular}{llllll}
\hline Factor & Category & Positive/examined (\%) & OR & $95 \% \mathrm{Cl}$ & \multicolumn{1}{c}{$P$ value } \\
\hline Province & Henan & $277 / 536(51.68)$ & - & - & $0.8042-1.889$ \\
& Xinjiang & $58 / 102(56.86)$ & 1.233 & - & - \\
Season* & Spring & $20 / 155(12.90)$ & - & 3.3870 \\
& Winter & $61 / 133(45.86)$ & 5.719 & $3.200-10.22$ & $P<0.0001$ \\
& Autumn & $254 / 347(73.20)$ & 3.224 & $2.127-4.886$ & $P<0.0001$ \\
\hline
\end{tabular}

OR Odds ratio;

u*" indicates significant difference. 


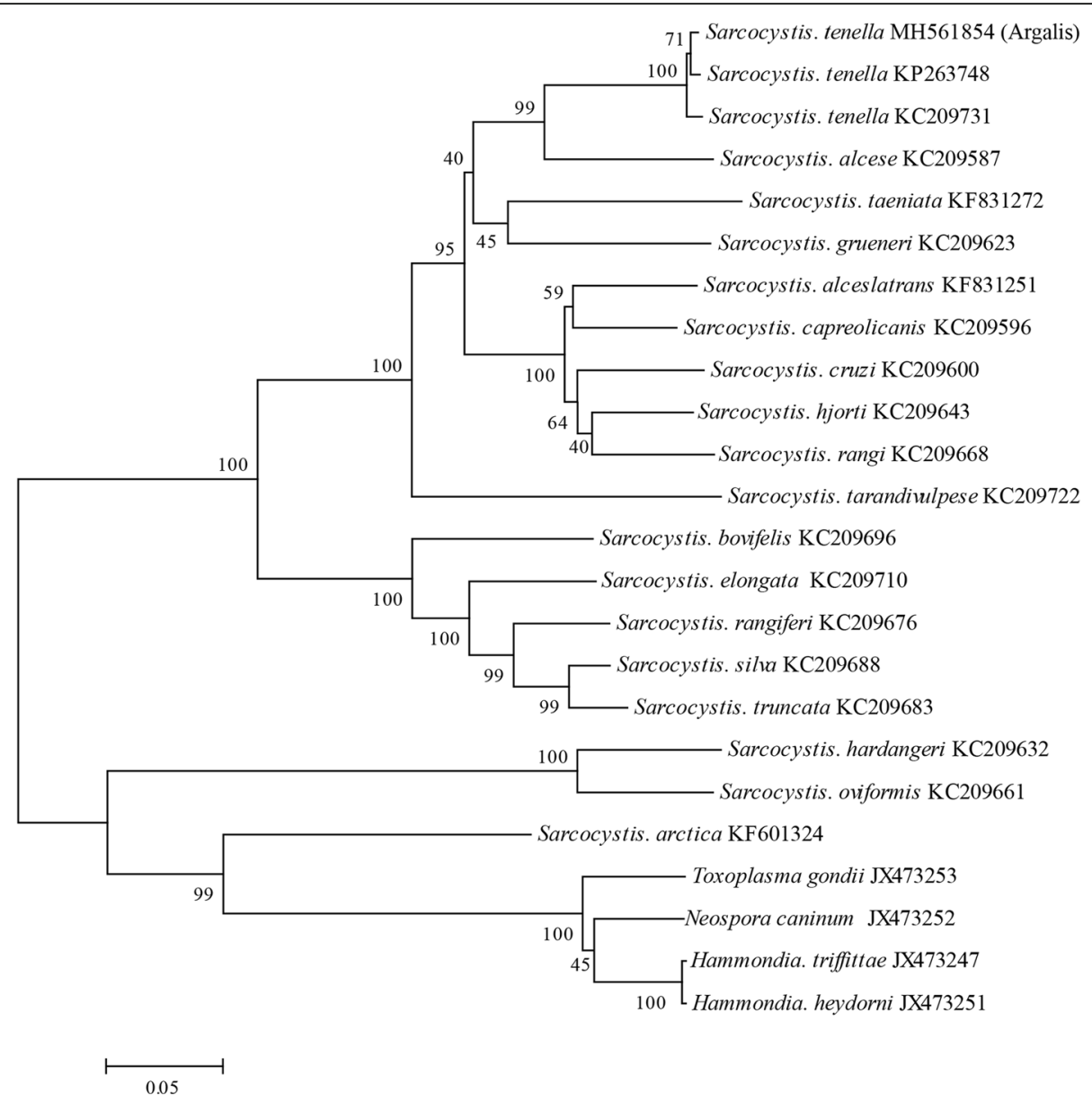

Fig. 2 Phylogenetic tree among the Sarcocystis tenella from argali identified in this study and other Sarcocystis spp. The phylogeny was inferred from neighbor-joining analysis of the mitochondrial cox1 sequences based on distances calculated using the Kimura two-parameter model. Bootstrap values $N>50 \%$ from 1000 replicates are shown at the nodes. Sarcocystis spp. (MH561854) is closely related to S. tenella (KP263748 and KC209731), indicating that argalis maybe intermediate hosts for S. tenella

sequences placed the sequence of $S$. tenella within a clade comprising the sequence of S. tenella (KP263748 and KC209731).

\section{Discussion}

The determination of the prevalence value for sarcocystosis in sheep depends on the method of detection. The method of using squashed tissue is fast and simple; however, the sensitivity is inferior to methods using tissue sectioning and PCR [2]. Usually, the detection ratio of Sarcocystis infection can be improved two-fold in swine by examining histological sections rather than squashed muscle [8]. Bradyzoites have been detected in pepsin-digested samples that had previously been found free of cysts using the squashed muscle method. The pepsin digestion method combined with PCR assays may be more sensitive than other methods for detecting Sarcocystis species [8]. Therefore, the $52.51 \%$ prevalence of Sarcocystis infection found in this study of domestic sheep may be lower than the real value.

Sarcocysts have been reported in the diaphragm, esophagus, heart, and skeletal muscles of domestic sheep and goats in China. Most reports of Sarcocystis in sheep have been published in Chinese. In this study, the prevalence of sarcocystosis was higher than the national average in China (41.52\%) [3]. It was also higher than the corresponding values from Asia $(48.02 \%, 26,061 / 54270)$, Europe (31.42\%, 3158/10051), and South America $(36.22 \%, 343 / 947)$, but lower than the prevalence of sarcocystosis in Oceania (93.31\%, 990/1061), Africa (54.67\%, 621/1136), and North America $(67.40 \%, 1141 /$ 1693) $[2,3]$. In this study, the prevalence of sarcocysts in sheep was high (52.51\%), but the prevalence of myositis was low (3.45\%). The results indicate that in most cases, the sarcocysts are non-pathogenic for the sheep. 
S. gigantea and $S$. medusiformis are two species with macroscopic cysts [2]. S. gigantea has not been found in the heart but occurs primarily in the esophagus, larynx and tongue muscles [9]. S. medusiformis cysts are found primarily in the diaphragm, abdominal muscles, and carcasses [2]. We did not find macroscopic sarcocysts in any of the sheep heart samples examined; this is in accordance with the distribution of Sarcocystis in host tissues described by Dubey et al. [2]. In our study, the sarcocysts in heart muscles were identified as cysts of $S$. tenella and $S$. arieticanis by morphology, location, host, and molecular characteristics. The average sarcocysts loading in muscle from domestic sheep was $18.07 \pm 29.87$ cysts per square centimeter, which is much higher than that in beef $\left(1.40 \mathrm{cysts} / \mathrm{cm}^{2}\right)$ and feral pigs $\left(3.03 \mathrm{cysts} / \mathrm{cm}^{2}\right)$ from the U.S. and mutton $\left(7.84 / \mathrm{cm}^{2}\right)$ from China $[8,10$, 11]. The higher parasite load indicates that the domestic sheep we examined had contact with an environment severely contaminated with sporocysts or the specific strains. Although most Sarcocystis species are non-pathogenic for sheep, infection may have an adverse impact on the health of domestic sheep, may endanger meat quality, and may lead to economic losses for the livestock industry.

Both Henan Province (located in central China) and Xinjiang Province (located in the northwest of China) had a high prevalence of Sarcocystis infection, indicating that Sarcocystis may be widespread in China. Usually, sarcocysts appear in striated muscle 1 2 month post-ingestion of sporocysts. The risk of Sarcocystis infection in the autumn was higher than that in winter or spring $(P<0.01)$ (Table 2$)$. It has been speculated that summer and autumn may pose a higher risk for infection with Sarcocystis sporocysts compared with winter or spring. Few epidemiological studies have assessed the risk factors associated with Sarcocystis in sheep. However, it has been hypothesized that contact with canids or felids, a moist environment, and age are risk factors for Sarcocystis [2].

Sarcocystis species have strict host-genus specificity, and they infect many wild and domestic ruminant animals [2]. S. ferovis was found in Bighorn sheep, but it was not transmissible to domestic sheep [12]. However, S. cruzi was present in both domestic cattle and wood bison [13]. A Sarcocystis species was first identified from wild sheep (Ovis musimon) [14], and $S$. tenella and $S$. arieticanis were later identified from European Mouflon (Ovis ammon musimon) [15]. In this study, only microscopic sarcocysts were found in argali.

The 18S rRNA gene, the 28S rRNA gene and the internal transcribed spacer 1 (ITS1) were employed to identify the molecular characteristics of Sarcocystis spp.
Gjerde reported that $\operatorname{cox} 1$ is a useful genetic marker for Sarcocystidae [16], because it is helpful to resolve the unclear species boundaries of closely related Sarcocystis spp. in different hosts. $\mathrm{Hu}$ et al. reported that the $18 \mathrm{~S}$ rRNA, 28S rRNA, and cox1 genes for S. tenella shared high identities with those of S. capracanis, i.e., 99.0, 98.3, and $93.6 \%$, respectively $[17,18]$. Cox 1 seemed to perform better than $18 \mathrm{~S}$ rRNA or $28 \mathrm{~S}$ rRNA for distinguishing $S$. tenella from $S$. capracanis [16]. The sequence of sarcocysts from argali (MH561854) was submitted to GenBank. The phylogenetic analysis based on near full-length mitochondrial $\operatorname{cox} 1$ sequences placed the sequence of $S$. tenella within a clade including the sequences of S. tenella (KP263748 and KC209731) (Fig. 2). This work is the first report concerning the Sarcocystis infection in argali from China, indicating that argali may be a natural intermediate host for $S$. tenella.

\section{Conclusion}

We found a high prevalence and parasite load of Sarcocystis in sheep from both central and northwestern China. We also examined two specimens of argali (Ovis ammon) and found sarcocysts in the myocardium of one animal. The sequence of the cox 1 gene of sarcocysts from argali suggested that the species was S. tenella. This report is the first to indicate that argali may be a natural intermediate host for $S$. tenella.

\section{Methods}

\section{Sampling of naturally infected animals}

From March 2014 to October 2017, we examined a total of 638 domestic sheep hearts (all animals over six months old) from selected areas in Henan and Xinjiang provinces for Sarcocystis infection (Fig. 3, Table 1). We also evaluated samples from the hearts and diaphragms of two argalis (males, 2-3 years old) for the occurrence of sarcocysts. The argalis came from zoos in 2017 and 2018, where they had died from diarrhea. All samples were transported to the Laboratory of Veterinary Pathology, Henan Agricultural University (Zhengzhou, Henan, China) in cooler boxes. All samples were examined for macroscopic and microscopic cysts of Sarcocystis.

\section{Microscopic examination}

Muscles were cut from each sheep heart and squashed between two glass slides, then examined microscopically at $\times 10$ magnification for the presence of sarcocysts. Three pieces of muscle from each sample were fixed in $10 \%$ formalin in a neutral buffer for paraffin sections $(5 \mu \mathrm{m})$ and were stained with $\mathrm{H} \& \mathrm{E}$ (hematoxylin-eosin) and IHC (immunohistochemistry) reagents. Rabbit anti-Toxoplasma gondii polyclonal antibody was kindly provided by Dr. Dubey (ARS, 

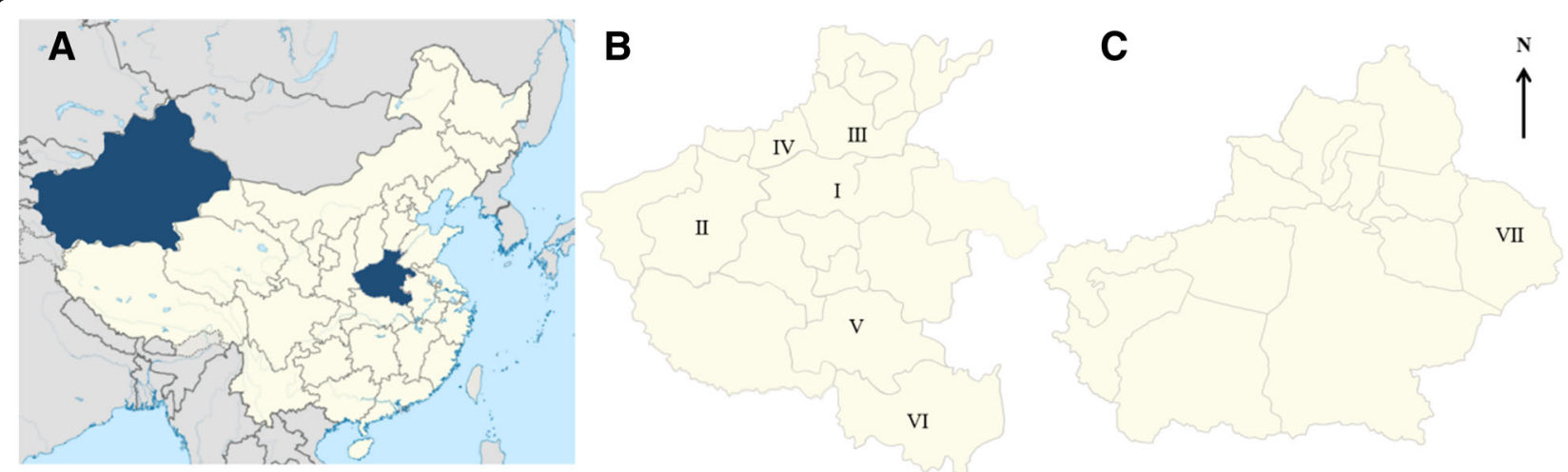

Fig. 3 Map showing the location of samples received from Henan and Xinjiang provinces in China. Map adapted from Google Earth. a Location of Henan and Xinjiang provinces in China; b Areas of collection within Henan Province. I: Zhengzhou, II: Luoyang, III: Xinxiang, IV: Jiaozuo, V: Zhumadian, VI: Xinyang; c Area of collection within the Xinjiang Uygur Autonomous Region. VII: Hami

USDA). A mouse- and rabbit-specific 3, 3'-diaminobenzidine- horseradish peroxidase (HRP/DAB) Avidin-biotin complex $(\mathrm{ABC}) \mathrm{IHC}$ detection kit was purchased from Abcam (ab64264). The sarcocysts were observed and photographed under a Leica DFC320 light microscope.

\section{Pepsin digestion examination}

Individual samples of the myocardium $(50 \mathrm{~g})$ were homogenized and digested in acidic pepsin [19]. The digested tissues were examined for bradyzoites and cysts of Sarcocystis species by light microscopy.

\section{Molecular identification}

Individual sarcocysts were isolated from the muscle of sheep and argalis under a stereomicroscope. DNA was extracted from single sarcocysts using a commercial DNA extraction kit (Tiangen Biotec Company, DP304, China). PCR was performed to amplify a segment of the mitochondrial cytochrome c oxidase subunit 1 gene ( $\operatorname{cox} 1)$ using the specific primer pairs of SF1 and SR9. The amplified PCR product was approximately $1038 \mathrm{bp}[16,18,20]$. The amplified PCR products of sarcocysts were sent to Beijing Nuosai Biological Engineering Biotechnology Company for bi-directional sequencing on an ABI PRISM ${ }^{\mathrm{mi}} 3730 \mathrm{XL}$ DNA Analyzer using the BigDye Terminator v3.1 Cycle Sequencing Kit (Applied Biosystems, Foster City, CA, USA). The obtained sequences were analyzed by BLAST. MEGA 6.0 software and was used to construct a phylogenetic tree of the S. tenella isolates using the neighbor-joining method (Kimura two-parameter model). Bootstrap analysis using 1000 replicates was used to assess the robustness of clusters.

\section{Statistical analysis}

Statistical analysis was performed with the GraphPad Prism 4.0 software (GraphPad Software Inc., San Diego,
CA, USA). The data were analyzed by a Chi-square test or Fisher's exact test to determine the association between infection with Sarcocystis and risk factors such as location (Xinjiang and Henan provinces) and seasons (spring, autumn, and winter). $P<0.05$ was considered statistically significant.

\section{Abbreviations \\ cox1: Mitochondrial cytochrome c oxidase subunit 1 gene; H\&E: Hematoxylin and eosin staining; IHC: Immunohistochemistry; PCR: Polymerase chain reaction}

\section{Acknowledgements}

We would like to thank Yongjie Feng, Yaoyao Lu, Kai Wang, Mengyao Wang, Feng Cheng and Yafei Liu (Henan Agricultural University, Zhengzhou, China) for collecting samples.

\section{Funding}

The China Postdoctoral Science Foundation (2016 M600577) and the Program for Science and Technology Innovation Talents in Universities of Henan Province (17HASTIT038) supported this study, including taking of samples, purchasing reagents and paying researchers.

\section{Availability of data and materials}

The datasets used and/or analyzed during the current study are available from the corresponding author upon reasonable request.

\section{Authors' contributions}

HD performed the data analysis and wrote the manuscript. RJS, YHW, ZXT helped in collecting samples. LXZ and JJH helped in the revision of the manuscript. YRY designed the experiment and wrote the manuscript. All authors have read and approved the final version of the manuscript.

\section{Ethics approval and consent to participate}

Verbal consent for collecting samples from the farm animals and zoo was obtained. The methods employed are widely used in China and were approved by the ethics committee of Henan Agricultural University (China). The protocol was approved by the Beijing Association for Science and Technology (SYXK [Beijing] 2007-0023).

Consent for publication Not applicable 


\section{Competing interests}

The authors declare no competing interests. None of the authors of this report have financial or personal relationships with other people or organizations that could inappropriately influence its content.

\section{Publisher's Note}

Springer Nature remains neutral with regard to jurisdictional claims in published maps and institutional affiliations.

\section{Author details}

${ }^{1}$ College of Animal Science and Veterinary Medicine, Henan Agricultural University, Zhengzhou 450002, China. ${ }^{2}$ Center for Animal Disease Control and Prevention of Henan Province, ZhengZhou 450002, China. ${ }^{3}$ School of Biological Sciences, Yunnan University, Kunming 650091, China.

\section{Received: 10 September 2018 Accepted: 23 November 2018}

Published online: 03 December 2018

\section{References}

1. Dubey JP. Lesions in sheep inoculated with Sarcocystis tenella sporocysts from canine feces. Vet Parasitol. 1988;26(3-4):237-52.

2. Dubey JP, Calero Bernal R, Rosenthal BM, Speer CA. Fayer. Sarcocystosis of animals and humans. 2nd Edn. Boca Raton: CRC Press; Taylor \& Francis Group; 2016.

3. Dong H, Lu YY, Yang YR. Epidemiplpgy and classification of Sarcocystis in sheep and goat. Chin J Zoonoses. 2017;33:828-36 in Chinese.

4. Dubey JP, van Wilpe E, Calero-Bernal R, Verma SK, Fayer R. Sarcocystis heydorni, n. sp. (Apicomplexa: Sarcocystidae) with cattle (Bos taurus) and human (Homo sapiens) cycle. Parasitol Res. 2015;114(11):4143-7.

5. Blagojevic B, Antic D. Assessment of potential contribution of official meat inspection and abattoir process hygiene to biological safety assurance of final beef and pork carcasses. Food Control. 2014;36:174-82

6. Tenter AM. Current research on Sarcocystis species of domestic animals. Int J Parasitol. 1995;25(11):1311-30.

7. Collins GH, Atkinson E, Charleston WAG. Studies on Sarcocystis species III: the macrocystic species of sheep. N Z Vet J. 1979;27(10):204-6.

8. Calero-Bernal R, Verma SK, Oliveira S, Yang Y, Rosenthal BM, Dubey JP. In the United States, negligible rates of zoonotic sarcocystosis occur in feral swine that, by contrast, frequently harbour infections with Sarcocystis miescheriana, a related parasite contracted from canids. Parasitology. 2015; 142(4):549-56.

9. Gual I, Bartley PM, Katzer F, Innes EA, Canton GJ, Moore DP. Molecular confirmation of Sarcocystis gigantea in a naturally infected sheep in Argentina: a case report. Vet Parasitol. 2017;248:25-7.

10. Pritt B, Trainer T, Simmons-Arnold L, Evans M, Dunams D, Rosenthal BM. Detection of Sarcocystis parasites in retail beef: a regional survey combining histological and genetic detection methods. J Food Prot. 2008;71(10):2144-7.

11. Dong H, Lu YY, Wang YH, Feng YJ, Dang SY, Liang HD, Yang YR. Histopathology and typing of Sarcocystis in myocardium of cattle and sheep. Chin J Vet Sci. 2018;38(1):160-4.

12. Dubey JP. Sarcocystis ferovis sp $n$ from the Bighorn Sheep (Ovis canadensis) and Coyote (Canis latrans). Proc. Helminthol. Soc. Wash. 1983;50(1):153-8.

13. Calero-Bernal R, Verma SK, Seaton CT, Sinnett D, Ball E, Dunams D, Rosenthal BM, Dubey JP. Sarcocystis cruzi infection in wood bison (Bison bison athabascae). Vet Parasitol. 2015;210(1-2):102-5.

14. Nigro M, Mancianti F, Rossetti P, Poli A. Ultrastructure of the cyst and life cycle of Sarcocystis sp. from wild sheep (Ovis musimon). J Wildl Dis. 1991; 27(2):217-24.

15. Odening K, Stolte M, Walter G, Bockhardt I. Cyst wall ultrastructure of two Sarcocystis spp. from European mouflon (Ovis ammon musimon) in Germany compared with domestic sheep. J Wildl Dis. 1995;31(4):550-4

16. Gjerde B. Phylogenetic relationships among Sarcocystis species in cervids, cattle and sheep inferred from the mitochondrial cytochrome c oxidase subunit I gene. Int J Parasitol. 2013;43(7):579-91.

17. Hu JJ, Liu T, Liu Q, Esch GW, Chen JQ, Huang S, Wen T. Prevalence, morphology, and molecular characteristics of Sarcocystis spp. in domestic goats (Capra hircus) from Kunming, China. Parasitol Res. 2016. 115(10):3973-81.
18. Hu JJ, Huang S, Wen T, Esch GW, Liang Y, Li HL. Sarcocystis spp. in domestic sheep in Kunming City, China: prevalence, morphology, and molecular characteristics. Parasite. 2017; 24:30.

19. Dubey JP. Toxoplasmosis of animals and humans. 2nd ed. Boca Raton: CRC Press; Taylor \& Francis Group; 2010.

20. Gjerde B. Sarcocystis species in red deer revisited: with a redescription of two known species as Sarcocystis elongate n. sp. and Sarcocystis truncata n. sp. based on mitochondrial cox1 sequences. Parasitology. 2014:141(3):441-52

\section{Ready to submit your research? Choose BMC and benefit from:}

- fast, convenient online submission

- thorough peer review by experienced researchers in your field

- rapid publication on acceptance

- support for research data, including large and complex data types

- gold Open Access which fosters wider collaboration and increased citations

- maximum visibility for your research: over $100 \mathrm{M}$ website views per year

At $\mathrm{BMC}$, research is always in progress.

Learn more biomedcentral.com/submissions 